\title{
A Concept of Mobile Support System for the Young Onset Dementia in Taiwan
}

\author{
Ming-Hsiang TU, RN, PhD ${ }^{\mathrm{a}}$, Yung-Hsiang TU, $\mathrm{PhD}^{\mathrm{b}}$, \\ Huey-Jane LEE, RN, $\mathrm{MS}^{\mathrm{c}}$ and Polun CHANG, $\mathrm{PhD}^{\mathrm{d}}$ \\ a Assistant Professor, School of Nursing, National University of \\ Nursing and Health Sciences, Taipei, Taiwan \\ ${ }^{\mathrm{b}}$ Assistant Professor, Department of Industrial Design, \\ Tatung University, Taipei, Taiwan \\ 'Assistant Secretary General, Taiwan Alzheimer's \\ Disease Association, Taipei, Taiwan \\ ${ }^{\mathrm{d}}$ Institute of BioMedical Informatics, National \\ Yang-Ming University, Taipei, Taiwan
}

\begin{abstract}
Owing to the increasing population of young onset dementia all over the world. We designed a 6 "-display Android mobile system for evaluating the potential patient of Dementia. A pilot test at experienced nursing members showed that they had strong willing of launching this design in their daily practical events.
\end{abstract}

Keywords. young onset dementia, Android-based design, mobile device, technology acceptance model

\section{Introduction}

The WHO announced a global action plan on the health response to Dementia from 2017 to 2025. In the plan, countries like Taiwan, the number of patients with Dementia are mentioned to be highly climbed. According to the investigation of Taiwan Alzheimer's Disease Association (TADA), 2019, at the group of age above 65, the patient rate is now $7.59 \%$ [1]. The younger onset Dementia (YOD) who is normally age under 60, has lots mobility in their daily life, and who might have some multipart activities with others. They sometimes found that has forget something newly happens. They seldom admit his situation to be a Dementia or potentially being a patient of this disease. So, we need a quick identification tool for finding the potential patient and verifying this patient by his situation no matter at hospital or other occasion.

We have developed a set of interface design principles [2] to effectively design a complex documentation with 6"-display Android mobile system, based on a TADA model [1], which is a form of multi-category with 15 pages assessment forms and assessment questions was more than 200. We have continuously used a David's Technology Acceptance Model (TAM) questionnaire to evaluate users' acceptance of 6"-display Android mobile system.

In this pilot study, we show the concept design of the interface of the YOD system and gives some important evaluation for it, including the user demand among the family members, hospital staffs, and other helpers. 


\section{Methods}

A validated TAM questionnaire [3] to evaluate the 6"-display Android mobile system that consisted of 12 easy to use, 9 usefulness, and 15 satisfaction of question. The 6"display Android mobile system has four categories of main items: Status, Contact, Demand, and Preparation in Figure 1 displays the main interface items are located at the left of the Android device screen. There are some different sub-items show in the front of screen for user to check and record. At the below of the screen, there are also 4 items constantly display for user to see the evaluation score of the potential patient.

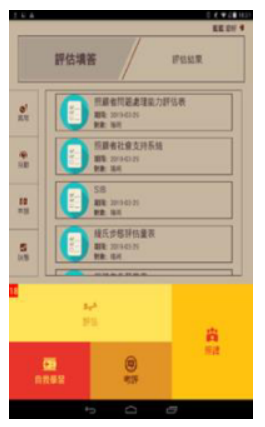

Figure 1. The young onset dementia system interface.

\section{Results}

The results of their opinions can be concluded at three parts. Firstly, the display and screen size of PDA was good enough for them to carry on and easy to use. Secondly, the user interface (UI) was easy to read. Lastly and most importantly one is that more than $50 \%$ of the pilot users said they are willing to use this new tool for their daily work.

\section{Conclusions}

The system is easy to use and can provide quality care plans for the younger of dementia. We are at the early stage of promoting this system and many dementia institutes are highly interested in testing.

\section{References}

[1] Dementia. (2019). Retrieved from http://www.tada2002.org.tw/About/IsntDementia

[2] Chang P, Hsu CL, Liou YM, Kuo YY, Lan CF. Design and development of interface design principles for complex documentation using PDAs. CIN: Computers, Informatics, Nursing. 2011; 29(3): 174-183.

[3] Tu MH, Chang P, YL L. (2018, Oct). Avoiding obsolescence in mobile health: Experiences in designing a mobile support system for complicated documentation at long-term care facilities. CIN: Computers, Informatics, Nursing, 2018 Oct; 36(10):501-506. 